\title{
Enseñar a leer en la Universidad, para desarrollar el pensamiento crítico
}

\section{Teaching to read in the University, for developing critical thinking}

\author{
Silvia C. Acosta-Velázquez ${ }^{a}$, Elba M. Pedraza-Amador ${ }^{b}$,Zaira L. González Gómez ${ }^{c}$
}

\begin{abstract}
:
Reading is not a simple decoding of printed symbols; it has effects on learning and leads to the construction of meanings. Considering that accessing a text does not guarantee comprehension and learning, reading comprehension has become a constant concern of the education system at all levels for more than two decades, due to it is one of the fundamental resources to achieve learning and for the performance of adult life in different fields. The purpose of this article is to present the results of a review of publications around reading, focusing on the low reading proficiency of students, prior to their admission to higher education, as well as the skills they need to acquire during their vocational training to increase their opportunities to enter the labor market of the knowledge society with higher chances of success. It is also analyzed, from the perspective of different authors, the role of teacher as promoters of the development of reading competence and critical thinking, through the application of reading strategies in the activities of the higher education level.
\end{abstract}

Keywords:

Reading, reading comprehension, critical thinking, written text

\section{Resumen:}

La lectura no es una simple decodificación de símbolos impresos, tiene efectos en los aprendizajes y conduce a la construcción de significados. Considerando que el hecho de acceder a un texto no garantiza la comprensión y el aprendizaje, la comprensión lectora se ha convertido en una preocupación constante del sistema educativo en todos los niveles desde hace más de dos décadas, debido a que es uno de los recursos fundamentales para lograr los aprendizajes y para el desempeño de la vida adulta en diferentes ámbitos. El propósito de este artículo es presentar los resultados de una revisión de las publicaciones en torno a la lectura, centrándose en la baja competencia lectora de los estudiantes, previo a su ingreso a la educación superior, así como en las competencias que requieren adquirir durante su formación profesional para incrementar sus oportunidades de ingresar al mercado laboral de la sociedad del conocimiento con mayores probabilidades de éxito. Se analiza también, bajo la perspectiva de diferentes autores, el papel del docente como promotor del desarrollo de la competencia lectora y el pensamiento crítico, mediante aplicación de estrategias de lectura en las actividades propias del nivel de educación superior.

\section{Palabras Clave:}

Lectura, Comprensión lectora, Pensamiento crítico, texto escrito

\section{Introducción}

A lo largo de su vida, el ser humano hace uso de diferentes recursos para su aprendizaje y para adquirir habilidades, destrezas y conocimientos, entre ellos la lectura, reconocida como práctica social que da inicio en edades tempranas, a la par de la inserción formal al sistema educativo, y que se mantiene, de manera permanente, como uno de los instrumentos para continuar los aprendizajes más allá del ámbito escolar y en la vida adulta (Marbá, Márquez \& Sanmartí, 2009).

Leer, en su definición más simple refiere el acto de "pasar la vista por lo escrito" (RAE, 2019) para comprender su significado o hacer una interpretación, sin embargo, lograr la comprensión implica el establecimiento de una

Autor de Correspondencia, Universidad Autónoma del Estado de Hidalgo, https://orcid.org/0000-0001-8972-7748, Email: silvia_acosta2066@uaeh.edu.mx

b Universidad Autónoma del Estado de Hidalgo, https://orcid.org/0000-0002-7182-2437, Email: elbam@uaeh.edu.mx

c Universidad Autónoma del Estado de Hidalgo, https://orcid.org/0000-0003-1688-2581, Email: go354615@uaeh.edu.mx 
relación autor-texto-lector (Oliveras \& Sanmartí, 2009) y la aplicación de habilidades superiores del pensamiento, es decir, de los procesos de análisis, síntesis y evaluación de la información, por lo que la lectura de textos académicos y otros materiales en que se apoya el proceso de enseñanza-aprendizaje, si bien contribuye al cumplimiento de los requisitos en los diferentes niveles educativos, no garantiza el avance académico de los estudiantes ni otorga la certeza de que ha comprendido lo que lee y está en capacidad de usar la información para construir nuevos saberes.

Las dificultades en la comprensión de la lectura son un asunto pendiente en el ámbito de la educación, con mayor relevancia en los niveles medio superior y superior, dada la necesidad de utilizar textos técnicos para la elaboración de trabajos escolares y de investigación. Esto representa un obstáculo en el proceso de aprendizaje de los estudiantes, que se agrava ante las dificultades para la interpretación de textos y elaboración de críticas, debido a la carencia de habilidades lectoras y la escasa capacidad para el análisis, síntesis y evaluación de la información (OECD, 2000, 2012; Hernández y Cols., 2012; Reis y Cols., 2001) lo cual limita sus posibilidades de insertarse exitosamente al mercado laboral, en función de los retos que impone la actual sociedad del conocimiento.

En este sentido, el Programa para la Evaluación Internacional de Alumnos (PISA, por sus siglas en inglés) de la Organización para la Cooperación y el Desarrollo Económicos (OCDE) introducido con el propósito de evaluar los conocimientos y habilidades adquiridos por los estudiantes en la etapa final de la escolaridad obligatoria (quince años) necesarios para su plena participación en la sociedad del conocimiento (Márquez, 2017), se centra en la medición del desempeño y la capacidad de resolución de problemas mediante la aplicación de conocimientos en las áreas de lectura, matemáticas y ciencias en el contexto internacional (OCDE, 2006a).

Para efectos de la evaluación de las aptitudes para lectura, PISA hace uso de tres escalas, otorgando puntajes a los estudiantes en función de su respuesta a reactivos con diferentes grados de dificultad, a partir de lo cual establece una clasificación de cinco niveles de dominio:

a) Dominio en el nivel 5, caracterizado por la capacidad de hacer inferencias, evaluar críticamente los textos y manejar información en textos con los cuales no están familiarizados b) Dominio en el nivel 4, en donde el estudiante es capaz de evaluar críticamente un texto e interpretar significados a partir de sutilezas del lenguaje

c) Domino en el nivel 3, de complejidad moderada, caracterizado por la vinculación de distintas partes de un texto y la capacidad para relacionarlo con conocimientos previos

d) Dominio del nivel 2, en el cual los estudiantes únicamente son capaces de reconocer ideas sencillas y ubicar información directa

e) Dominio del nivel 1, propio de estudiantes que no son capaces de realizar la lectura básica que mide la prueba, lo cual sugiere una limitante a la adquisición de conocimientos (OCDE, 2006a).

Las estadísticas de la OCDE muestran qué en el año 2000, más de la mitad de los estudiantes evaluados a nivel internacional se ubicaban en el nivel 2 o mayor, en tanto que, en México en el mismo año, el $44 \%$ de los alumnos participantes de la prueba obtuvieron resultados de dominio del nivel 1.

Para el año 2018, los resultados del desempeño de los estudiantes mexicanos no mostraron cambios significativos, reportándose el $1 \%$ de estudiantes con dominio de nivel 5 , mientras que el $35 \%$ no logró el nivel mínimo de competencia, es decir el nivel de dominio 2, en ninguna de las tres áreas evaluadas (OECD, 2019).

Dada la relación entre la comprensión lectora y el aprendizaje (Solé, 2012), en función de que la mayor parte de las actividades en el contexto escolar sientan sus bases en ella, los datos anteriores son evidencia de una limitada capacidad para alcanzar mayores niveles de razonamiento y apuntan a que la lectura que hacen los estudiantes próximos a ingresar al nivel medio superior sólo promueve la reproducción de ideas a través de la memorización y la repetición, pero no conduce a la construcción de significados sobre lo leído (Salinas, Diez y López, 2009) al no existir una evaluación de los contenidos del texto, para lograr su comprensión.

Considerando el insuficiente nivel de comprensión lectora de los estudiantes a su ingreso a la educación superior y para efectos de destacar la relevancia del rol del docente en el proceso de desarrollo de competencias que favorecen el pensamiento crítico y la reflexión, en este trabajo se realizó una exhaustiva revisión de la literatura disponible en torno a dicho fenómeno, la información obtenida fue sometida a análisis con el propósito de generar las conclusiones plasmadas en el documento. 


\section{Comprensión lectora y prácticas de lectura}

Al entenderse como capacidad esencial para el desarrollo de los individuos a nivel mundial y lograr su participación en la sociedad (Rovira y López, 2017), la lectura es una de las prioridades de PISA (Ministerio de Educación, 2010), destacándose la necesidad de que las nuevas generaciones adquieran habilidades y competencias básicas que contribuyan a la concreción de sus aprendizajes, es decir, que desarrollen la competencia lectora (Monroy \& Gómez, 2009), reconociéndose a esta no únicamente como un componente del aprendizaje de los estudiantes, sino como requisito para desempeñarse exitosamente en distintas áreas de su vida adulta, definiéndose como "la capacidad de comprender, utilizar y analizar textos escritos para alcanzar los objetivos del lector, desarrollar sus conocimientos y posibilidades de participar en la sociedad" (OCDE, 2006), o bien como "la capacidad de un individuo para comprender, emplear, reflexionar e interesarse en los textos escritos con el fin de lograr sus metas personales, desarrollar sus conocimientos, su potencial personal y, en consecuencia, participar en la sociedad" (INEE, 2012), sin embargo, diversos estudios en relación a la comprensión lectora tanto en México como en otros países de Latinoamérica (Cornejo, Roble, Barrero \& Martín, 2012; Hernández, Rodríguez \& Vargas, 2012) apuntan a que es una de las mayores debilidades del sistema educativo; la casi nula competencia lectora de los estudiantes obstaculiza el proceso de aprendizaje a lo largo de su formación, y tiene repercusiones en su desempeño al ingresar al nivel de educación superior.

En México, estas deficiencias y los bajos niveles alcanzados por los alumnos en el área de comprensión lectora, dieron lugar a la implementación de estrategias para mejorar su rendimiento académico y contribuir a su formación integral, a través de la inclusión de programas para el desarrollo de la competencia lectora en los niveles previos a la educación superior; entre ellos el Programa Nacional para el Fortalecimiento de la Lectura y Escritura (PRONALEES), el Programa Nacional de Lectura (PNL), y el Programa de Fomento para el Libro y la Lectura: México Lee (SEP, 2008; SEP, 2017), entre otros, que sin embargo, no han tenido el resultado esperado, en tanto que la comprensión de los textos académicos y científicos propios de la educación superior suponen, todavía, un alto grado de dificultad para los alumnos.

En este sentido, algunos autores proponen que el lector haga uso de diferentes estrategias para lograr la comprensión de distintos textos (Solé, 2012; Muñoz \& Ocaña, 2017; García, Arévalo \& Hernández, 2018; Sardá, Márquez \& Sanmartí, 2006), de tal forma que active sus conocimientos previos para construir otros nuevos. Se destaca así mismo, la relevancia de la variabilidad textual como estrategia para comprender los textos utilizados con fines académicos, en tanto que "favorecen el desarrollo de habilidades de razonamiento y de pensamiento crítico" (Cornejo, Roble, Barrero \& Martín, 2012, p.156) y coadyuvan la comprensión, señalando como ejemplo de esto, la inclusión de tópicos de historia y cultura en programas educativos relacionados con ciencia y tecnología.

Respecto a este tópico, se precisa destacar que en la actual sociedad, inmersa en procesos de globalización, acelerados cambios tecnológicos, manejo de información y constante renovación del conocimiento, la lectura sigue siendo considerada como una actividad académica a la cual se recurre para el abordaje de los contenidos de las asignaturas y la participación en clase, sin tomar en cuenta la conveniencia de la incorporación de textos de diversos géneros y el uso de estrategias cognitivas que favorezcan el desarrollo de habilidades de razonamiento y pensamiento crítico para contribuir a la mejora en la comprensión de conceptos, el análisis y la formación de juicios a partir de la toma de conciencia de las cuestiones sociales y las necesidades humanas, requisito insoslayable para la práctica de las distintas disciplinas.

La lectura, señala Ruffinelli (2011), es tratada como parte importante de los procesos de alfabetización en todos los niveles educativos con propósitos meramente utilitarios, sin que se le reconozca como un proceso interactivo de comunicación que además de conducir a la interpretación y construcción de significados, contribuye a ampliar el vocabulario y el reconocimiento de términos que más adelante permitirán organizar, analizar y evaluar la información, favoreciendo la reflexión y la apropiación de conocimientos.

Sin embargo, en la sociedad actual, donde los conocimientos se renuevan de manera constante y se requiere la transformación de la información en conocimientos significativos útiles en los entornos económico, social y laboral, no se propicia la lectura como un elemento para el descubrimiento y el enriquecimiento (Reyzábal \& Tenorio, 2004), ni se considera el valor que ésta tiene para participar de manera efectiva y exitosa en los diferentes ámbitos, mediante el ejercicio de una profesión que atienda las cuestiones sociales y las necesidades de las personas (Cornejo \& Cols., 2012) en respuesta a las exigencias del mundo contemporáneo.

Para esclarecer lo anterior, conviene mencionar que en la educación superior la lectura es una actividad mecanizada que conduce a la extracción de información 
concreta contenida en un texto, sin embargo, el "saber leer" como menciona Ruffinelli (2012) no consiste en un proceso alfabetizador donde prevalece la visualización de símbolos y palabras y la reproducción de ideas, sino en el entendimiento de lo que se lee, y que por tanto, impide a los estudiantes de nivel superior la elaboración de críticas y la interpretación de textos técnico-didácticos de uso común para sus actividades escolares.

Al respecto, el origen de las dificultades para el análisis y comprensión de textos parece ser producto de los escasos o nulos hábitos de lectura, la ausencia de un propósito para la lectura, el desconocimiento de métodos para leer y la pobreza en el dominio del lenguaje (Salinas, Diez \& López, 2009), en tanto que en los libros de texto y materiales didácticos proliferan términos técnicos y científicos específicos con los que los alumnos no están familiarizados, y constituyen una limitante para el aprendizaje al carecer de significado para ellos, con la consecuente afectación a su formación académica y profesional, sin embargo, partiendo del hecho que enseñar a leer es un proceso a cargo del docente en el nivel inicial de educación, el papel de éste resulta fundamental en la educación superior, toda vez que puede convertirse en guía de los estudiantes en el proceso de asimilación del conocimiento mediante la implementación de estrategias y prácticas lectoras que conduzcan a la transformación de información en aprendizajes significativos.

\section{La lectura en la universidad}

Se asume que al ingresar a la educación superior, los alumnos conocen las estrategias adecuadas para abordar los diferentes tipos de lectura y cuentan con el dominio del lenguaje y las habilidades suficientes para la comprensión de textos académicos (Vidal \& Márquez, 2016), sin embargo, en investigaciones realizadas en México y otros países de Latinoamérica se destaca el bajo nivel de competencia lectora de los estudiantes (Cárdenas, 2014; Garrido, 2014; García, Arévalo \& Hernández, 2018) reconociéndose que existe una deficiencia en cuanto a los métodos de lectura empleados en los niveles educativos previos (Méndez, Espinal, Arbeláez, Gómez \& Serna, 2014), lo que impide a los estudiantes alcanzar los niveles de razonamiento requeridos para su formación universitaria y su posterior desempeño en la sociedad.

Considerando que la competencia lectora es fundamental para alcanzar el desarrollo de otras competencias que hoy en día son una exigencia para enfrentar los problemas de un mundo globalizado (Flores, 2016), se hace énfasis en la promoción de la lectura en la universidad, en el establecimiento de estrategias de comprensión de diversas tipologías textuales y en el desarrollo de habilidades para comprender las formas del lenguaje propias de este nivel educativo, propiciando así el desarrollo personal, social y profesional de los educandos. Pese a ello, la responsabilidad continúa recayendo en el estudiante-lector (López, 2013), quien visualiza la lectura como una necesidad y una obligación académica, con fines prácticos para su formación (Martos, Campos, Figarés \& Quiles, 2015).

Aunado a esto, ante las dificultades de los estudiantes para la comprensión de textos académicos, Cornejo y cols. (2012) refieren la simplificación de las demandas por parte del profesorado, que orienta las prácticas lectoras a la identificación de conceptos explícitos y a la extracción de información mediante una lectura literal, que lejos de favorecer el hábito de la lectura y el desarrollo de la competencia lectora, desmotiva a los alumnos y en poco apoya al proceso de aprendizaje y a la producción de conocimiento en el contexto de la sociedad actual (Avendaño, 2016).

En relación a lo anterior, debe tenerse en cuenta que, como menciona Flores (2016), para lograr el rendimiento académico de los alumnos y mejorar "su desempeño intelectual y cognitivo en todos los aspectos de la vida" (p.132), la lectura es necesaria no sólo en los niveles de educación básica, sino también en la educación superior, por lo que ésta debe asumir entre sus objetivos prioritarios el desarrollo del pensamiento crítico (Méndez y cols., 2014), tendiente a lograr el acceso de los estudiantes a los conocimientos propios de su disciplina y su formación como profesionales competentes para la resolución de problemas asociados a la productividad y competitividad de las empresas en la sociedad contemporánea.

Para lograr tal propósito, se requiere fomentar en ellos una cultura lectora, a la vez que reafirmar sus aprendizajes mediante la utilización de estrategias de lectura y cognitivas que los conduzcan a interpretar y elaborar críticas sobre los textos utilizados como apoyo para la transmisión de los contenidos de sus asignaturas, es decir, al estudiante universitario se le debe enseñar el proceso de comprensión lectora (Campos, 2007), con la finalidad de que identifique la información relevante en los textos de su disciplina y a partir de su interpretación, esté en posibilidades de elaborar conclusiones respecto a lo leído.

Evidentemente, transformar la información en conocimientos significativos con utilidad concreta en el entorno económico, social y laboral no es una tarea fácil, 
dada la rapidez con que se produce la información, se renuevan los conocimientos y se desarrolla la tecnología en la actualidad, además de las ya mencionadas dificultades de comprensión lectora relacionadas al rendimiento escolar en la educación superior, la falta de claridad en los propósitos de la lectura y el escaso apoyo por parte de los docentes (García, Arévalo \& Hernández, 2018).

Por otra parte, debe considerarse que en la educación superior no se cuenta con indicadores de lectura que permitan medir el desempeño de los estudiantes y que, como refieren Salado, Ramírez-Martinell \& Ochoa (2017), las prácticas lectoras en el aula se orientan casi exclusivamente a la extracción de contenidos relacionados con la disciplina, y en ese sentido, los alumnos reciben influencia por parte de sus profesores, lo que repercute en su trayectoria académica y limita por tanto, su desempeño en el ámbito laboral a futuro.

\section{El docente universitario y el desarrollo de la competencia lectora}

Si bien es cierto que en la educación superior la lectura constituye una de las herramientas fundamentales para acceder al conocimiento, en las instituciones no existe homogeneidad respecto a los modos de leer de los estudiantes, las demandas de los profesores, ni las técnicas didáctico-pedagógicas que se utilizan para favorecer el desarrollo de la competencia lectora, es decir, por lo general la enseñanza de la lectura no se encuentra presente en el proceso formativo universitario (Cárdenas, 2014). Se asume, como una idea preconcebida que los estudiantes no leen, y se anticipa su conducta de renuencia a la lectura con fundamento en las deficiencias en el área reportadas en los exámenes de ingreso, así como en las estadísticas internacionales relacionadas con el desempeño en tres áreas concretas: lectura, matemáticas y ciencias (OECD, 2019).

Se atribuyen sus dificultades de comprensión a una formación inadecuada y deficiencias no superadas en niveles educativos previos, a los escasos hábitos lectores y al desconocimiento de estrategias de lectura (Cardona, Osorio, Herrera \& González, 2018) sugiriéndose además la falta de tiempo y la ausencia de motivación e inducción a la lectura por parte de los docentes, asumiéndose que no consideran la relevancia de la competencia lectora en la formación profesional debido a que en este nivel educativo, es común que los requisitos exigidos para impartir cátedra se orienten a la experiencia y la formación disciplinar, y no a la formación pedagógica que les otorgue las bases para apoyar su quehacer docente y el desarrollo de estrategias específicas para conducir a los estudiantes a la comprensión e interpretación de textos académicos (Vidal \& Manríquez, 2016) de utilidad para la elaboración de trabajos de investigación (Flores, 2016).

Al respecto, si como se señaló anteriormente, las dificultades en la comprensión lectora provienen de deficiencias no resueltas en niveles previos, debe considerarse también que en la educación superior las exigencias de habilidades lectoras y los propósitos de lectura son completamente diferentes en función de las diversas disciplinas, lo que obliga al uso de nuevas formas y estrategias para leer, y por tanto, a la reconfiguración del papel del docente en el proceso de enseñanza-aprendizaje.

Se manifiesta entonces la necesidad de enseñar a leer en la universidad, no para soslayar las deficiencias de los estudiantes, sino como una oportunidad para desarrollar el pensamiento crítico mediante estrategias de comprensión lectora (Solé, 2007) que incentiven los procesos reflexivos y de evaluación de la información, y favorezcan la asimilación de nuevos conocimientos a partir de los saberes previos (Avendaño, 2016), toda vez que la lectura es una herramienta de utilidad no sólo para aprender, sino también para pensar, al conformarse, junto con la escritura, en un instrumento para estructurar el pensamiento (Solé, Mateos, Miras, Castells, Cuevas \& Gracia, 2005). Es decir, a través de la lectura y la escritura, los individuos pueden operar las ideas, transformarlas, producir nuevos conocimientos y desarrollar el pensamiento (Serrano, 2014), por lo que, en el nivel de educación superior, se implica hoy en día, una revisión del proceso formativo del profesorado y sus prácticas docentes, dado el papel fundamental que cumplen en el proceso de enseñanza aprendizaje.

En síntesis, "saber leer" o entender lo que se lee (Ruffinelli, 2012) ha dejado de ser una tarea exclusiva del alumno, para convertirse en una responsabilidad de las instituciones de educación superior y sus docentes, que incluye, además de la identificación de las necesidades de aprendizaje de los estudiantes, la motivación por la lectura y el esclarecimiento de su propósito (Flores, 2016), la propuesta de tareas de lectura que favorecen los aprendizajes (Serrano, 2014), y el diseño, selección y aplicación de métodos de enseñanza y estrategias que contribuyan a lograr los objetivos que su formación disciplinar plantea (INEE, 2012).

\section{CONCLUSIONES}

La lectura como instrumento para el aprendizaje en el entorno académico tiene un valor indiscutible, al ser un medio para acceder a la información y a la adquisición de 
conocimientos. En este sentido, se destaca la estrecha relación existente entre lectura y comprensión lectora y la presencia de dos escenarios respecto al tema: aprender a leer y leer para aprender.

Si bien es cierto que se esperaría que al concluir la educación media superior los alumnos hayan alcanzado un nivel de dominio lector más allá de la reproducción de contenidos, es un hecho que a su ingreso a la educación superior se caracterizan por sus escasas habilidades para la búsqueda, análisis, síntesis y evaluación de la información necesaria para el desarrollo de las actividades escolares propias de ese nivel, con repercusiones graves en su proceso de aprendizaje y en el rendimiento escolar.

De acuerdo a las investigaciones realizadas en el contexto internacional, existe una gran cantidad de variables que determinan la baja competencia lectora, sugiriéndose que además del desarrollo insuficiente de habilidades lecto-escritoras en los niveles de educación básica y media superior, los escasos hábitos lectores, la falta de motivación y la ausencia de un propósito para la lectura, inciden directamente en la comprensión y en el desarrollo del pensamiento crítico, lo cual repercute en las capacidades de los individuos para hacer frente a las exigencias del mercado laboral actual, inmerso en la sociedad del conocimiento, que demanda la aplicación de tales competencias para la resolución de problemáticas y la adecuada toma de decisiones.

A pesar de la preocupación constante que esta problemática supone para el sistema educativo y la implementación de programas orientados al desarrollo de la competencia lectora, en México los resultados continúan apuntando a bajos niveles, sin cambios significativos desde hace dos décadas, por lo que esta competencia y las competencias asociadas siguen siendo una materia pendiente para las instituciones de educación superior, de quienes se precisa que planteen estrategias y establezcan medios para formar a su personal docente como lectores y mediadores de lectura. Sin embargo, es poco el avance en este campo. Los docentes orientan sus prácticas a la enseñanza de la disciplina concediendo a los textos una función transmisora de contenidos, sin que medie en el proceso la enseñanza de estrategias que favorezcan la comprensión y el desarrollo el pensamiento crítico, y a la vez motiven a los estudiantes a la creación de hábitos lectores.

En este sentido, es obligatorio propiciar la transformación del rol del alumno y conducirlo a una participación más activa en su proceso formativo, con lo que al pasar del acto mecánico de la lectura literal a una lectura crítica logrará la adquisición de conocimientos $y$, en consecuencia, tendrá mayores oportunidades para insertarse en el campo laboral y participar en la sociedad como ciudadano crítico y comprometido con el bien común.

Por último, debe considerarse que una de las funciones del docente, al margen de la disciplina de su especialidad, es promover la lectura en el aula, más allá del uso con fines académicos, y proveer al alumno de estrategias que le permitan la interpretación de lo leído y la construcción de significados, el desarrollo de sus capacidades, la familiarización con el proceso de comprensión lectora y la adquisición de conocimientos, lo que coadyuvaría el desarrollo de la competencia de pensamiento crítico desde el currículum, así como la transformación del aula universitaria en el espacio donde se genere la interlocución e interacción necesaria que requiere el proceso lector para lograr la introducción de los estudiantes a las particularidades de los campos disciplinares en los que se desenvuelve su orientación profesional.

En este orden de ideas y no obstante la falta de compromiso del docente universitario y la indiferencia de las instituciones de educación superior para promover la lectura observadas hasta hace poco, se precisa señalar el incremento en el número de universidades que han comenzado a adoptar el rol de mediadoras culturales y a través de actividades de extensión, propician espacios de reflexión para contribuir a la formación integral de los estudiantes.

Un claro ejemplo de ello son las 46 universidades de diferentes países agrupadas en la Red de Universidades Lectoras (RIUL), entre las que se cuentan 4 instituciones mexicanas: a) la Universidad Jesuita de Guadalajara (ITESO), b) la Universidad Nacional Autónoma de México (UNAM), c) la Universidad de Guadalajara y d) la Universidad Veracruzana.

Surgida en España en noviembre de 2006 con el objetivo principal de potenciar el papel de la lectura y la escritura en la Universidad, la RIUL promueve la formación integral de los estudiantes y el desarrollo de una visión crítica, a la vez que fomenta entre sus integrantes el uso de actividades diseñadas para la creación de entornos favorables a la lectura y la escritura en la universidad, el empleo de sus recursos humanos, materiales e institucionales para dinamizar los espacios para el aprendizaje, el debate y el diálogo, y la integración de nuevas vías para acceder a la información (RIUL, 2021). 
Como parte de las actividades de colaboración que la red promueve, en noviembre de 2014 estableció un convenio con el Instituto de Investigaciones Bibliotecológicas y de la Información (IIBI) de la Universidad Nacional Autónoma de México (UNAM) para el desarrollo del proyecto de Cartografía de lectura, escritura e información en las universidades mexicanas (UNAM, 2014), orientado a recabar información relacionada con las acciones, alcances y experiencias sobre lectura emprendidas por universidades mexicanas, así como las actividades de promoción y fomento a la lectura.

En el marco de las actividades mencionadas, resulta conveniente señalar la relevancia que adquieren las ferias del libro y destacar los esfuerzos que en el estado de Hidalgo realiza la Universidad Autónoma del Estado de Hidalgo (UAEH) para acercar a la comunidad universitaria y a sociedad en general a la lectura, a través de la Feria Universitaria del Libro (FUL), que tras 34 años consecutivos se encuentra posicionada entre las cinco mejores ferias del libro a nivel nacional y es un referente cultural.

Lo anterior es un ejemplo del rol fundamental de las universidades como promotoras de la lectura, y de las aportaciones que las actividades de extensión y la enseñanza de la lectura hacen al proceso formativo de profesionales, para acercarlos a su exitosa inserción al ámbito laboral, como individuos capaces de asumir una postura crítica frente a los retos que impone la sociedad del conocimiento.

\section{Referencias}

Avendaño, G. S. (2016). La lectura crítica en. Cuadernos de Lingüística Hispánica(28), 207-232. doi:http://dx.doi.org/10.19053/0121053X.4916

Campos, A. (2007). Pensamiento crítico . Bogotá: Cooperativa Editorial Magisterio .

Cárdenas, L. M. (2014). Prácticas de lectura y escritura en educación superior: lo que los estudiantes y docentes dicen de la lectura y la escritura en las licenciaturas de una universidad en el Caribe colombiano. Praxis, 10(1), 60-77. doi:https://doi.org/10.21676/23897856.1359

Cardona, S., Osorio, A., Herrera, A., \& González, J. M. (2018). Actitudes, hábitos y estrategias de lectura de ingresantes. Educación y Educadores, 21(3), 485-503. doi:10.5294/edu.2018.21.3.6

Cornejo, J., Roble, M. B., Barrero, C., \& Martín, A. M. (2012). Hábitos de lectura en alumnos universitarios de carreras de ciencia y de tecnología. Revista Eureka sobre Enseñanza y y Divulgación de la Ciencia, 9(1), 155-163. doi:DOI: 10498/14631 http://hdl.handle.net/10498/14631
Flores, D. (2016). La importancia e impacto de la lectura, redacción y pensamiento crítico en la educación superior. Zona Próxima, 128-135. doi:http://dx.doi.org/10.14482/zp.24.8727

García, M., Arévalo, M., \& Hérnández, C. A. (2018). La comprensión lectora y el rendimiento escolar. Cuadernos de Lingüística Hispánica(32), 155174. Obtenido de http://www.scielo.org.co/pdf/clin/n32/2346-1829clin-32-155.pdf

Garrido, F. (2014). Leer y escribir para ingresar a la Educación Superior. Revista de la Educación Superior, XLIII(4)(172), 145-150. Obtenido de http://publicaciones.anuies.mx/pdfs/revista/Revist a172_S4A1ES.pdf

Hernández, C., Rodríguez, N., \& Vargas, A. (2012). Los hábitos de estudio y motivación para el aprendizaje de los alumnos en tres carreras de ingeniería. Revista de la Educación Superior, XLI (3), (163), 67-87. Obtenido de http://www.scielo.org.mx/pdf/resu/v41n163/v41n1 63a3.pdf

INEE. (2012). Los textos contínuos ¿Cómo se leen?. La competencia lectora dese PISA. México: INEE.

López, Francesc (Coord.). (2013). Comprensión Lectora. El uso de la lengua como procedimiento. Barcelona: Graó-Colofón.

Marbá, A., Márquez, C., \& Sanmartí, N. (2009). ¿Qué implica leer en la clase de ciencias? Alambique. Didáctica de las Ciencias Experimentales(59), 102-111. Obtenido de http://gent.uab.cat/conxitamarquez/sites/gent.uab.c at.conxitamarquez/files/que $\% 20 \mathrm{implica} \% 20$ leer $\%$ 20en\%20clase \%20de\%20ciencias.pdf

Márquez, A. (2017). A 15 años de PISA: Resultados y polémicas. Perfiles Educativos, XXXIX(156), 3-15. Obtenido de https://www.redalyc.org/pdf/132/13250923001.pd $\mathrm{f}$

Martos, E., Campos, M., \& Quiles, C. (2015). En E. Ramírez, Tendencias de la Lectura en la Universidad (págs. 1-13). México: UNAM.

Méndez, J., Espinal, C., Arbeláez, D., Gómez, J., \& Serna, C. (2014). La lectura crítica en la educación superior: un estado de la cuestión. Revista Virtual Universidad Católica del Norte(41), 4-18. Obtenido de https://www.redalyc.org/articulo.oa?id=19422998 0002

Ministerio de Educación. (2010). La lectura en PISA 2009. Marcos y pruebas de la evaluación. Madrid: Secretaría General Técnica. Gobierno Español. Obtenido de https://www.educacionyfp.gob.es/inee/dam/jcr:82 853972-c574-4ce3-9ac4-8039237054c0/lecturaen-pisa.pdf

Monroy, J. A., \& Gómez, B. E. (2009). Comprensión lectora. Revista Mexicana de Orientación Educativa, Online. Obtenido de 
http://pepsic.bvsalud.org/scielo.php?script=sci_art text\&pid=S1665-75272009000100008

Muñoz, A., \& Ocaña, M. (2017). Uso de estrategias metacognitivas para la comprensión textual. Cuadernos de Lingüística Hispánica(29), 223244. Obtenido de https://www.redalyc.org/pdf/3222/322249834011. pdf

OCDE. (2006). El Programa PISA de la OCDE ¿Qué es y para qué sirve? París: OECD. Obtenido de https://www.oecd.org/pisa/39730818.pdf

OCDE. (2006). PISA 2006. Marco de la Evaluación. Conocimientos y habilidades en Ciencias, Matemáticas y Lectura. OCDE. Obtenido de https://www.oecd.org/pisa/39732471.pdf

OCDE. (2012). Programa para la Evaluación Internacional de Alumnos (PISA). PISA 2012 Resultados. México: OCDE. Obtenido de https://www.oecd.org/pisa/keyfindings/PISA2012-results-mexico-ESP.pdf

OECD. (2019). Programa Internacional de Evaluación de los Alumnos (PISA). PISA 2018 - Resultados. Obtenido de https://www.oecd.org/pisa/publications/PISA2018 _CN_MEX_Spanish.pdf

Oliveras, B., \& Sanmartí, N. (2009). La lectura como medio para desarrollar el pensamiento crítico. Educación Química, 233-245. Obtenido de http://www.scielo.org.mx/scielo.php?script=sci_ar ttext\&pid=S0187-893X2009000500005

RAE. (2019). Diccionario de la lengua española, 23. ${ }^{a} \mathrm{ed}$., [versión 23.3 en línea. Obtenido de Real Academia de la Lengua: https://dle.rae.es

Reis, J. C., Guerra, A., Braga, M., \& Freitas, J. (2001). History, Science and Culture: Curricular Experiences in Brazil. Science \& Education, 10(4), 369-378. doi:10.1023/A:1011289614116

Reyzábal, M. V., \& Tenorio, P. (2004). El aprendizaje significativo de la literatura (Cuarta ed.). Madrid: Editorial La Muralla, S.A.

RIUL. (2021). Red Internacional de Universidades Lectoras. Obtenido de https://universidadeslectoras.es/

Rovira, Y., \& López, E. (2017). La lectura en la enseñanza universitaria. Revista de Ciencias Médicas e Pinar del Rúio, 21(3), Versión Online. Obtenido de http://scielo.sld.cu/scielo.php?script=sci_arttext\&p $\mathrm{id}=\mathrm{S} 1561-31942017000300013$

Ruffinelli, J. (2012). Comprensión Lectora (Tercera ed.). México: Trillas.

Salado, L., Ramírez-Martinell, A., \& Ochoa, R. (2017). Hábitos de lectura y afinidad tecnológica de los estudiantes. Estudios Lambda. Teoría y práctica de la didáctica en lengua y literatura(2), 1-24. Obtenido de https://www.uv.mx/personal/albramirez/files/2015 /06/salado_ramirez_lambda-2017.pdf

Salinas, H., Díez, G., \& López, A. (2009). Impacto de los cursos de taller de lectura y redacción en los estudiantes de la DES-DAEH. Memoria

Electrónica del X Congreso Nacional de

Investigación Educativa, (págs. 1-17). Veracruz, México. Obtenido de

http://www.comie.org.mx/congreso/memoriaelectr onica/v10/pdf/area_tematica_05/ponencias/0732F.pdf

Sardá, A., Márquez, C., \& Sanmartí, N. (2006). Cómo promover distintos niveles de lectura de los textos de ciencias. Revista Electrónica de Enseñanza de las Ciencias, 290-303. Obtenido de http://reec.uvigo.es/volumenes/volumen5/ART5_ Vol5_N2.pdf

SEP. (2008). Programa de Fomento para el Libro y la Lectura: México Lee. México: SEP-

CONACULTA. Obtenido de

https://www.sep.gob.mx/work/models/sep1/Resou rce/103893/1/MexLee.pdf

SEP. (2017). Programa de Fomento para el Libro y la Lectura 2016-2018. México: SEP. Secretaría de Cultura. Obtenido de https://observatorio.librosmexico.mx/files/progra ma_de_fomento_para_el_libro.pdf

Serrano, S. (2014). La lectura, la escritura y el pensamiento. Función epistémica e implicaciones pedagógicas. Lenguaje, 1(42), 97-122. Obtenido de

http://www.scielo.org.co/pdf/leng/v42n1/v42n1a0 5.pdf

Solé, I. (2007). Estrategias de Lectura. Barcelona: Colofón. Solé, I. (2012). Competencia lectora y aprendizaje. Revista Iberoamericana de Educación(59), 43-61. Obtenido de https://rieoei.org/historico/documentos/rie59a02.p df

Solé, I., Mateos, M. M., Martín, E., Castells, N., Cuevas, I., \& Gracia, M. (2005). Lectura, escritura y adquisición de conocimientos en Educación Secundaria y Educación Universitaria. Infancia y aprendizaje, 3(28), 329-347. Obtenido de https://redined.mecd.gob.es/xmlui/handle/11162/3 5189

UNAM. (2014). Cartografía de lectura, escritura e información en las Universidades Mexicanas. Obtenido de Instituto de Investigaciones Bibliotecológicas y de la Información: http://iibi.unam.mx/eramirez/proyecto.php

Vidal, D., \& Manríquez, L. (2016). El docente como mediador de la comprensión lectora en universitarios. Revista de la Educación Superior, 95-118. Obtenido de http://publicaciones.anuies.mx/pdfs/revista/Revist a177_S3A4ES.pdf 